\title{
QUALIDADE DE VIDA DOS DOCENTES: UM ESTUDO DE CASO
}

\author{
Eduarda Lauck MACHADO \\ Aluna do Bacharelado em Ciência e Tecnologia/UFVJM - Campus II, Rodovia MGT 367, km 583, nº 5000, \\ Alto da Jacuba, Diamantina, MG, 39100-000. E-mail: eduarda.lauck@ hotmail.com \\ Paulo César de Resende ANDRADE \\ Professor Dr. do Instituto de Ciência e Tecnologia/UFVJM - Campus II, Rodovia MGT 367, $\mathrm{km}$ 583, $\mathrm{n}^{\circ}$ \\ 5000, Alto da Jacuba, Diamantina, MG, 39100-000. E-mail: paulo.andrade@ ict.ufvjm.edu.br \\ Cácia Aparecida CAMPOS \\ Assistente em Administração do Instituto em Ciência e Tecnologia/UFVJM - Campus II, Rodovia MGT \\ 367, km 583, nº 5000, Alto da Jacuba, Diamantina, MG, 39100-000. E-mail: cacia@ ict.ufvjm.edu.br \\ Lucas Cordeiro MACEDO \\ Aluno do Bacharelado em Ciência e Tecnologia/UFVJM - Campus II, Rodovia MGT 367, km 583, $\mathrm{n}^{\circ} 5000$, \\ Alto da Jacuba, Diamantina, MG, 39100-000. E-mail: lucascm.macedo@ gmail.com \\ Valéria Cláudia Ferreira ANDRADE \\ Aluna de Especialização/UFVJM - Campus II, Rodovia MGT 367, km 583, n 5000, Alto da Jacuba, \\ Diamantina, MG, 39100-000. E-mail: valeria.andrad@ hotmail.com
}

\begin{abstract}
Resumo: A qualidade de vida (QV) é um conceito ligado ao desenvolvimento humano. Não significa apenas que o indivíduo ou o grupo social tenham saúde física e mental, mas que estejam bem com eles mesmos, com a vida, com as pessoas que os cercam, enfim, ter qualidade de vida é estar em equilíbrio. A importância da avaliação da qualidade de vida vem ganhando crescente valorização tanto no meio acadêmico, quanto na sociedade, e esse fenômeno vem sendo amplamente reconhecido nas últimas décadas. O objetivo deste trabalho foi analisar como está a qualidade de vida de docentes da UFVJM, em Diamantina, buscando verificar a associação entre qualidade de vida e as variáveis demográficas, relacionadas ao exercício da profissão, e auto-percepção do estado de saúde e da qualidade de vida. Foi estudada uma amostra de 79 docentes em 2010 por meio da aplicação do questionário WHOQOL/breve e calculado a média e o desvio-padrão para os escores deste questionário de QV. O escore médio apresentado pelos docentes na avaliação da QV foi de 69,59 pontos. O domínio "meio ambiente" mostrou-se o mais afetado, apresentando a menor média de escores, enquanto o "físico", com maior valor, apresentou-se como aspecto positivo da qualidade de vida dos docentes. Verificou-se que, $81 \%$ dos docentes consideraram sua QV boa ou muito boa, uma vez que estão satisfeitos ou muito satisfeitos com relação a este aspecto.
\end{abstract}

Palavras-chave: Qualidade de vida. Docentes. Domínios. Saúde. Satisfação.

\begin{abstract}
The quality of life (QOL) is a concept linked to human development. Not only means that the individual or social group have physical and mental health, but that are good about themselves, about life, people around them, finally, to have quality of life is to be in balance. The importance of evaluating the quality of life is gaining increasing importance both in academia, society, and this phenomenon has been widely recognized in recent decades. The objective of this study was to analyze how is the quality of life of teachers from UFVJM in Diamantina, seeking to verify the association between quality of life and demographic variables related to the profession, and self-perception of health status and quality of life. The sample consisted of 79 teachers in 2010. We used the WHOQOL/brief and calculated the mean and standard deviation for the scores of the QOL questionnaire. The mean score made by the teachers in the assessment of QOL was 69.59 points. The domain "environment" proved to be the most affected, with the lowest average scores, while the "physical", with greater value, presented as a positive quality of life of teachers. It was found that $81 \%$ of teachers considered their QOL good or very good, once they are satisfied or very satisfied with this regard.
\end{abstract}

Keywords: Quality of life. Teachers. Domains. Health. Satisfaction. 


\section{INTRODUÇÃO}

Qualidade de vida, conceito ligado ao desenvolvimento humano, implica estar em equilíbrio (Bowling e Brasier, 1995). Para garantir uma qualidade de vida satisfatória é necessário ter hábitos saudáveis, tempo para lazer, cuidar bem do corpo e manter outros hábitos que façam o indivíduo se sentir bem, proporcionando conseqüências apropriadas como, usar o humor para lidar com situações de estresse, definir objetivos de vida e, o principal, sentir controle sobre a própria vida.

Segundo Spitzer (1987), entende-se por qualidade de vida (QV) a percepção do indivíduo tanto de sua posição na vida, no contexto da cultura e nos sistemas de valores nos quais se insere, como em relação aos seus objetivos, expectativas, padrões e preocupações. O conceito de QV é amplo uma vez poder ser afetado de modo complexo por diversos fatores entre os quais pela saúde física do indivíduo, pelo seu estado psicológico, por suas relações sociais, por seu nível de independência e pelas suas relações com as características mais relevantes do meio ambiente.

Medir QV é bastante complexo pelo fato de não encontrar definição consensual sobre o que ela realmente significa, entretanto, existe razoável concordância entre os pesquisadores acerca do constructo QV, cujas características são: subjetividade, multidimensionalidade e bipolaridade. De acordo com Sptizer (1987), o pesquisador deverá definir em seu estudo ou em seu trabalho de investigação o que está considerando como QV ou, então, estabelecer em qual definição esse conceito se operacionaliza. Os pesquisadores devem delinear claramente o que entende por $\mathrm{QV}$ e identificar os diversos domínios a serem avaliados, considerando que cada domínio identifica um foco particular de atenção e agrupa vários itens (Gill, 1994).

A Organização Mundial de Saúde (OMS) desenvolveu um instrumento (questionário) para aferir a $\mathrm{QV}$, designado de WHOQOL (World Health Organization Quality of Life) que possui duas versões validadas para o português, o WHOQOL 100, composto por 100 questões e, o WHOQOL - Breve, composto por 26 questões. O WHOQOL - 100 é constituído por seis domínios: o físico, o psicológico, o do nível de independência, o das relações sociais, o do meio ambiente e o dos aspectos religiosos. O WHOQOL - Breve é constituído por quatro domínios: o físico, o psicológico, o das relações sociais e o do meio ambiente (Fleck et al, 2000).

A avaliação da QV vem ganhando importância crescente nas últimas décadas, tanto no meio acadêmico quanto na sociedade e, portanto, o tema tem despertado o interesse de pesquisadores, em diversas áreas do conhecimento, a saber: doenças crônicas e 
efeitos de medicamentos (Bonicatto et al, 2001; Fleck et al, 2002; Cahan et al, 2003); enfermagem (Saupe et al, 2004); fisioterapia (Gravina, 2002); odontologia (Silva e Rosa, 1996; Logan et al, 1997; Leggat et al, 2001; Lewczuk et al, 2002; Nunes e Freire, 2006); sociologia e medicina (Bowlin e Brazier, 1995); gerontologia (Queiroz e Trinca, 1983; Chaimowicz, 1997; Ferraz e Peixoto, 1997; Costa et al, 2000; Diogo, 2003; Joia et al, 2007) e educação (Penteado e Pereira, 2007).

Segundo Penteado e Pereira (2007), o estudo da QV tem sido apontada como uma categoria analítica central para promover abordagens integradoras e interdisciplinares.

Neste sentido, não há dúvida de que o desenvolvimento de instrumentos e de formas de avaliação da QV ou do bem-estar é de suma importância mas, também, um grande desafio.

Tendo em vista a falta de estudos sobre QV de docentes vinculados ao serviço público federal e as mudanças ocorridas no mundo do trabalho que podem influenciá-la, o objetivo desta pesquisa foi descrever e avaliar os aspectos associados à QV dos profissionais da Universidade Federal dos Vales do Jequitinhonha e Mucuri (UFVJM), na cidade de Diamantina MG; considerando os domínios, físico, psicológico, relações sociais e com o meio ambiente, buscando verificar a associação da QV com as variáveis demográficas relacionadas ao exercício da profissão, por intermédio da autopercepção destes.

\section{MATERIAIS E MÉTODOS}

A amostra selecionada baseou-se no conjunto de docentes da UFVJM em exercício no segundo semestre de 2010, em Diamantina.

A base de dados primária utilizada para a construção do plano amostral foi fornecida pela Superintendência de Recursos Humanos - SRH/UFVJM, contendo informações sobre os docentes da UFVJM (nome do docente, unidade de lotação) no segundo semestre de 2010, que formaram a base primária de dados para a construção do plano amostral.

As estimativas obtidas apresentaram erro amostral máximo de $10 \%$, com nível de significância de 5\%. O tamanho da amostra foi calculado de maneira a atingir as estimativas desejadas. Foi utilizado um fator de correção de população finita no cálculo do tamanho da amostra. Inicialmente calculou-se o tamanho da amostra supondo população infinita seguindo a equação:

$$
\eta_{0}=\left(\frac{1,96}{2 \times 0,10}\right)^{2}=96,04 \approx 96
$$

Posteriormente foi calculada a correção de população finita: 


$$
\eta=\frac{\eta_{0}}{1+\frac{\eta_{0}-1}{N}}=\frac{96}{1+\frac{96-1}{320}}=74,02 \approx 74
$$

A pesquisa foi realizada entre agosto e outubro de 2010 perante aos docentes da UFVJM, em Diamantina/MG. A amostra participante da pesquisa foi significativa, uma vez que houve docentes de todos os cursos da universidade.

Dos 320 docentes da universidade, muitos não estavam em atividade no período da coleta de dados, pois, estavam inativos, de férias ou afastados (licenças médicas, maternidade, prêmio e por interesse particular). Ainda assim, participaram da pesquisa 79 docentes, que está acima do tamanho amostral calculado de 74.

A escolha metodológica orientou-se no sentido de contemplar a subjetividade do funcionário como eixo primordial no levantamento dos dados; daí a opção por instrumentos disponíveis na literatura que permitissem aos sujeitos se auto-avaliar, a partir de suas percepções.

Foi utilizado como instrumento de coleta de dados um questionário autoaplicável (Fleck et al, 2000), contendo o instrumento de qualidade de vida da OMS, na sua versão abreviada, WHOQOL-Breve. O WHOQOL-Breve contém 26 perguntas que envolvem aspectos diversos da vida cotidiana, das quais 24 são distribuídas em quatro domínios:
1) Físico: percepção do indivíduo sobre sua condição física. Contém as facetas: dor e desconforto; energia e fadiga; sono e repouso; atividades da vida cotidiana; dependência de medicação ou de tratamentos; capacidade de trabalho;

2) Psicológico: percepção do indivíduo sobre sua condição afetiva e cognitiva, cujas facetas são: sentimentos positivos; pensar, aprender, memória e concentração; auto-estima; imagem corporal e aparência; sentimentos negativos; espiritualidade/religião/ crenças pessoais;

3) Relações sociais: percepção do indivíduo sobre os relacionamentos sociais e os papéis sociais adotados na vida, com as seguintes facetas: relações pessoais; suporte (apoio) social; atividade sexual; e

4) Meio ambiente: percepção do indivíduo sobre aspectos diversos relacionados ao ambiente onde vive. Contém as facetas: segurança física e proteção; ambiente no lar; recursos financeiros; cuidados de saúde e sociais: disponibilidade e qualidade; oportunidades de adquirir novas informações e habilidades; 
participação em, e oportunidades de recreação/lazer; ambiente físico: (poluição / ruído / trânsito / clima); transporte.

Para cada aspecto da qualidade de vida expresso no questionário WHOQOL-Breve, o sujeito pode apresentar sua resposta por meio de escores que variam de um a cinco (escala Likert), sendo a condição pior no escore um e a melhor, no escore cinco. Os resultados dos domínios apresentam valores entre zero e cem, sendo piores os mais próximos de zero e melhores, os mais próximos de cem. Dessa forma, um sujeito que apresente valor igual a 50 para determinado domínio, pode ser considerado mediano para esse domínio (Penteado e Pereira, 2007).

$\mathrm{O}$ cálculo dos domínios padronizados do WHOQOL-breve segue a expressões definidas em função das perguntas constantes no questionário (WHOQOL, 1998).

Cada domínio é representado por várias facetas e suas questões foram formuladas com escala de intensidade (nada extremamente), capacidade (nada completamente), freqüência (nunca - sempre) e avaliação (muito insatisfeito - muito satisfeito; muito ruim - muito bom).

Além dos quatro domínios, o instrumento apresenta duas questões gerais, sendo que uma se refere à autopercepção da qualidade de vida e a outra sobre satisfação com a saúde (Logan et al, 1997). Ainda segundo esses autores, o WHOQOL-breve não prevê conceitualmente utilizar do escore global de qualidade de vida, então é calculado o escore de cada domínio, quanto mais alto o escore, melhor a qualidade de vida naquele domínio.

Depois de coletados, os dados foram tabulados e foi utilizado o programa estatístico SPSS para as análises e para o cálculo da média e do desvio-padrão, considerando os escores padronizados dos domínios do protocolo WHOQOL-Breve. A comparação entre os gêneros foi feita pelo teste de Wilcoxon (Penteado e Pereira, 2007).

$\mathrm{O}$ instrumento de coleta de dados e o Termo de Consentimento Livre e Esclarecido do Sujeito - TCLE foram submetidos à apreciação e aprovação pelo Comitê de Ética em consonância com a Resolução 196/1996 do Conselho Nacional de Saúde, que estabelece normas éticas para pesquisas com seres humanos. Todos os participantes assinaram voluntariamente o TCLE, tendo sido informados de que a pesquisa não ofereceria nenhum tipo de risco dos pontos de vista físico e psicológico.

\section{RESULTADOS E DISCUSSÃO}

Quanto aos resultados referentes às duas questões gerais do WHOQOL-Breve e à autopercepção do estado de saúde verificou-se que, $81 \%$ dos docentes consideraram sua QV boa ou muito boa, uma vez estarem satisfeitos 
ou muito satisfeitos com relação a este aspecto.

A maior parte dos entrevistados $(77,2 \%)$ considerou sua condição atual de saúde como boa ou muito boa, tendo sido apontado ainda, por 92,4\%, pouca necessidade de algum tratamento médico para desempenharem suas atividades.

O escore médio apresentado pelos docentes na avaliação da qualidade de vida (WHOQOL-Breve) foi de 69,59 pontos. $\mathrm{Na}$ Tabela 1 estão apresentados a média e o desvio-padrão para os escores padronizados dos domínios da QV.

Tabela 1 - Média e desvio-padrão dos escores dos domínios de qualidade de vida (WHOQOL/breve) de docentes da UFVJM, Diamantina, MG, 2010

\begin{tabular}{ccc}
\hline $\begin{array}{c}\text { Domínio da Qualidade de } \\
\text { Vida (WHOQOL/Abreviado) }\end{array}$ & Média & $\begin{array}{c}\text { Desvio- } \\
\text { padrão }\end{array}$ \\
\hline Físico & 73,3 & 14,9 \\
Psicológico & 72,3 & 13,1 \\
Relações Sociais & 70,3 & 16,4 \\
Meio Ambiente & 62,3 & 12,0 \\
\hline
\end{tabular}

Fonte: Estudo da qualidade de vida de docentes da UFVJM, 2010.

Observa-se, por meio da análise da média dos escores obtidos em cada domínio da $\mathrm{QV}$, que a maior variação em termos de desvio-padrão ocorreu no domínio "relações sociais" e a menor, no domínio "meio ambiente". O domínio "meio ambiente" mostrou-se o mais afetado, uma vez ter apresentado a menor média de escores $(62,3$;
DP $=12,0)$, enquanto que o "físico" com maior valor $(73,3$; DP $=14,9)$, apresentou-se como o aspecto mais positivo da QV dos docentes da UFVJM.

As Tabelas 2 e 3 apresentam os aspectos que se destacaram, respectivamente, tanto negativa como positivamente, cujos dados foram obtidos a partir da análise descritiva de cada uma das questões do WHOQOL-Breve.

Tabela 2 - Descrição dos aspectos da qualidade de vida (QV) dos docentes da UFVJM que se destacam de maneira negativa, com porcentagem de respostas obtidas.

Aspectos da QV avaliados negativamente

\begin{tabular}{|c|c|}
\hline Aspecto & $\%$ \\
\hline $\begin{array}{c}\text { Insatisfação com o acesso aos serviços de } \\
\text { saúde }\end{array}$ & 27,84 \\
\hline Insatisfação com o sono & 18,98 \\
\hline Falta oportunidade de lazer & 17,72 \\
\hline $\begin{array}{c}\text { Insatisfação com o meio de transporte que } \\
\text { possui }\end{array}$ & 16,45 \\
\hline $\begin{array}{c}\text { Insatisfação com as condições do local } \\
\text { onde mora }\end{array}$ & 15,18 \\
\hline
\end{tabular}

Fonte: Estudo da qualidade de vida de docentes da UFVJM, 2010.

Os resultados mostram que os aspectos que mais afetaram negativamente o grupo estudado relacionam-se à dificuldade de acesso aos serviços de saúde, lazer e moradia. 
Tabela 3 - Descrição dos aspectos da qualidade de vida (QV) dos docentes da UFVJM que se destacam de maneira positiva, com porcentagem de respostas obtidas.

\begin{tabular}{|c|c|}
\hline \multicolumn{2}{|l|}{ Aspectos da QV avaliados positivamente } \\
\hline Aspecto & $\%$ \\
\hline Capacidade de atribuir sentido à vida & 88,60 \\
\hline $\begin{array}{l}\text { Capacidade de locomoção } \\
\text { (boa e muito boa) }\end{array}$ & 86,07 \\
\hline $\begin{array}{l}\text { Dor física que impede atividades (nada ou } \\
\text { muito pouco) }\end{array}$ & 79,74 \\
\hline $\begin{array}{l}\text { Dependência médica na vida diária (nada } \\
\text { ou muito pouco) }\end{array}$ & 78,48 \\
\hline Capacidade de concentração & 77,21 \\
\hline
\end{tabular}

Fonte: Estudo da qualidade de vida de docentes da UFVJM, 2010.

Por outro lado, os aspectos que mais afetaram positivamente relacionam-se à capacidade de atribuir sentido à vida privada/doméstica/pessoal e as questões físicas.

No tocante à aparência física Domínio Físico - observa-se que a maioria (em torno de $73 \%$ ) considera como muito boa e aceita com tranquilidade a condição atual, mostrando um bom grau de auto-aceitação que é um aspecto fundamental para uma qualidade de vida adequada. A maioria dos docentes mencionou estar satisfeita com a capacidade para o trabalho, e ter energia suficiente para as atividades diárias.

Em relação aos sentimentos negativos, a maioria $(75 \%)$ respondeu que sentimentos como mau humor, desespero, ansiedade e depressão acontecem com pouca frequência em suas atividades diárias e, por consequência, se sentem bastantes seguros no desenvolvimento destas. $\mathrm{O}$ que mais se destaca - Domínio Psicológico - é o fato de os docentes darem bastante sentido à vida, constituindo também, aspecto fundamental na qualidade de vida. Capacidade que se sobressaiu às demais.

O Domínio Relações Pessoais revelou que grande parte dos entrevistados considera estar satisfeito com esta dimensão (amigos, parentes, conhecidos, colegas), afirmando também estarem satisfeitos ou muito satisfeitos, com o apoio que recebem destas pessoas. Um percentual de $73,4 \%$ afirmaram estar satisfeitos ou bastante satisfeitos quanto à vida sexual, nível de satisfação considerado alto.

No que se refere ao ambiente físico Domínio Meio Ambiente - (clima, barulho, poluição, atrativos), 64,5\% dos docentes afirmaram que o ambiente em que estão inseridos é apenas medianamente saudável, classificando como baixa a oportunidade de atividade de lazer oferecida aos entrevistados. Outro ponto constatado foi a classificação da satisfação das necessidades atendidas frente à remuneração recebida como média e baixa, pela maioria dos docentes. Verificou-se ainda que a qualidade de determinados serviços como saúde e transporte, não foram considerados adequados por parte dos 
docentes, sendo estes responsáveis pelas piores pontuações no domínio.

No quesito saúde, $27,8 \%$ dos participantes disseram estar insatisfeitos com o acesso ao serviço. Quando perguntado aos docentes, quão satisfeitos estavam com o meio de transporte, $63 \%$ disse estarem satisfeitos com o acesso.

\section{CONCLUSÃO}

As diferenças das médias entre os gêneros não foram significativas $(\mathrm{p}>0,05)$ pelo teste de Wilcoxon, indicando não haver diferenças na QV entre homens e mulheres, dentro do grupo de docentes estudados.

A maioria dos entrevistados disse estar satisfeitos com sua saúde e, apesar de alguma dor física ou incômodo manifestado, isto não os impede de realizar as atividades diárias, mostrando pouca necessidade de intervenção (tratamento médico), o que caracteriza indicador positivo para a qualidade de vida.

Os profissionais pesquisados apresentaram menor QV nos domínios meio ambiente e relações sociais e maior nos domínios físico e psicológico. Quanto aos resultados referentes às duas questões gerais do WHOQOL-Breve e a autopercepção do estado de saúde verificou-se que, $81 \%$ dos docentes da UFVJM consideraram sua QV boa ou muito boa, uma vez estarem satisfeitos ou muito satisfeitos com relação a este aspecto.

\section{REFERÊNCIAS}

BONICATTO, S.C.; DEW, M.A.; ZARATIEGUI, R.; LORENZO, L.; PECINA. P. Adult outpatients with depression: worse quality of life than in other chronic medical diseases in Argentina. Soc Sci Med. 2001. Vol. 52: 911-919.

BOWLING, A. e BRAZIER, J. Quality of life in social science and medicine. Soc Sci ed. 1995. Vol. 41: 1337-1338.

CHAIMOWICZ, F. A saúde dos idosos brasileiros às vésperas do século XXI: problemas, projeções e alternativas. Rev Saúde Pública. 1997. Vol. 31: 184-200.

CHAN, G.W.; UNGVARI, G.S.; SHEK, D.T.; LEUNG DAGGER, J.J. Hospital and community-based care for patients with chronic schizophrenia in Hong Kong: quality of life and its correlates. Soc Psychiatry Psychiatr Epidemiol. 2003. Vol. 38: 196-203.

COSTA, M.E.L.; UCHOA, E.; GUERRA, H.L.; FIRMO, J.O.A.; VIDIGAL, P.G.; BARRETO, S.M. The Bambuí health and ageing study (BHAS): methodological approach and preliminary results of a population-based cohort study of the elderly in Brazil. Rev Saúde Pública. 2000. Vol. 34: 126-135.

DIOGO, M.J.D. Satisfação global com a vida e determinados domínios entre idosos com amputação de membros inferiores. Rev Panam Salud Publica. 2003. Vol. 13: 395399.

FERRAZ, A.F.; PEIXOTO, M.R.B. Qualidade de vida na velhice: estudo em uma instituição pública de recreação para idosos. Rev Esc Enferm USP. 1997. Vol. 31: 316338. 
FLECK, M.P.A; LOUZADA, S.; XAVIER, M.; CHACHAMOVICH, E.; VIEIRA, G.; SANTOS, L.; PINZON, V. Aplicação da versão em português do instrumento abreviado de avaliação de qualidade de vida "WHOQOL/breve". Rev. Saúde Pública. 2000. Vol. 34: 178-183.

FLECK, M.P.A.; LIMA, A.F.B.S.; LOUZADA, S.; SCHESTATSKY, G.; HENRIQUES, A.; BORGES, V.X. Associação entre sintomas depressivos e funcionamento social em cuidados primários à saúde. Rev Saúde Pública. 2002. Vol. 36: 431-438.

GILL, T.F. A critical appraisal of the quality of quality of life measurements. JAMA 1994. Vol. 272: 619-626.

GRAVINA, M.E.R. LER - Lesões por Esforços Repetitivos: uma reflexão sobre os aspectos psicossociais. Saúde Soc. 2002. Vol. 11(2): 1-18.

JOIA, L.C.; RUIZ, T.; DONALÍSIO, M.R. Condições associadas ao grau de satisfação com a vida entre a população de idosos. Rev Saúde Pública. 2007. Vol. 41(1): 131-138.

LEGGAT, P.A.; CHOWANADISAI, S.; KEDJARUNE, U.; KUKIATTRAKOON, B.; YAPONG, B. Health of dentists in southern Thailand. Int Dent J. 2001. Vol. 51: 348-352.

LEWCZUK, E.; AFFESKA-JERCHA, A.; TOMCZYK, J. Occupational health problems in dental practice. Med Pr. 2002. Vol. 53:

161-165.

LOGAN, H.L.; MULLER, P.J.; BERST, M.R.; YEANEY, D.W. Contributors to dentists job satisfaction and quality of life. $\mathrm{J}$ Am Coll Dent. 1997. Vol. 64(4): 39-43.

NUNES, M.F. e FREIRE, M.C.M. Qualidade de vida de cirurgiões-dentistas que atuam em um serviço público. Rev. Saúde Pública. 2006. Vol. 40 (6): 1019-1026.

PENTEADO, R.Z.; PEREIRA, I.M.T.B.

Qualidade de vida e saúde vocal de professores. Rev. Saúde Pública. 2007. Vol. 41(2): 236-243.

QUEIROZ, J.B.; TRINCA, S.F. A influência do lazer sobre pessoas da terceira dade. Rev Bras Enferm., RS. 1983. Vol. 36: 95-106.

SAUPE, R.; NIETCHE, E.A.; CESTAN, M.E.; GIORGI, M.D.M.; KRAHI, M. Qualidade de vida dos acadêmicos de enfermagem. Rev Latinoam Enfermagem. 2004. Vol. 12: 636-642.

SILVA, S.R.C.; ROSA, A.G.F. Características dos cirurgiões dentistas com vínculo empregatício em empresas públicas e/ou privadas no município de Araraquara-SP. Rev Odontol UNESP. 1996. Vol. 25: 9-18.

SPITZER, W.O. State of Science 1986: Quality of life and functional status as target variables for research. J Chronic Dis 1987. Vol. 40(6): 465-471.

WHOQOL Group. Development of the World Health Organization WHOQOL-Bref quality of life assessment. Psychol Med. 1998. Vol. 28: 551-558. 